OXFORD, A. E. (1958). J. gen. Microbiol. 19, 617-623

\title{
The Nutritional Requirements of Rumen Strains of Streptococcus bovis Considered in Relation to Dextran Synthesis from Sucrose
}

\author{
BY A. E. OXFORD \\ Plant Chemistry Division, Department of Scientific and Industrial Research, \\ Palmerston North, New Zealand
}

\begin{abstract}
SUMMARY: Five rumen strains of Streptococcus bovis all required biotin for good anaerobic production of dextran from sucrose in a simple acetate + bicarbonate + amino acids medium. Three of these strains required at least pantothenate also. When phosphate replaced acetate as a buffer, dextran production did not occur unless a soluble protein was also supplied in the medium.
\end{abstract}

Ford, Perry \& Briggs (1958) recently showed that not a single strain among 26 rumen strains of Streptococcus bovis required any B-vitamins for anaerobic growth in a glucose medium although, in agreement with previous observations of Niven, Washburn \& White (1948), they found that some of these strains appeared to require one or more B-vitamins when incubated aerobically. In view of the observations of Bailey \& Oxford $(1958 a, b)$, who showed that all of 16 rumen strains of $S$. bovis could produce good yields of dextran in a complex sucrose liquid medium only when certain cultural conditions were provided, it became of interest to determine whether these strains could ever produce dextran in a simplified protein-free medium devoid of all, or most, B-vitamins. The cultural conditions necessary for dextran production at $37^{\circ}$ were the provision of ample carbon dioxide and of a high concentration of phosphate or acetate. The nutrient basal medium used by Bailey \& Oxford was, however, complex, containing yeast extract and Casitone as sources of B-vitamins and organic nitrogen. With such a medium, strict anaerobiosis was neither necessary for, nor detrimental to, dextran production. The enzyme responsible for dextran production, dextransucrase, is secreted into the medium, and might conceivably need the provision of a large stabilizing colloid molecule for efficient action at $37^{\circ}$, since it is known that the Leuconostoc dextransucrase, secreted at $\mathbf{2 5}^{\circ}$, is not stable at $37^{\circ}$ (Bailey, Barker, Bourne \& Stacey, 1957). Nevertheless, it has proved possible to devise a simple medium for good dextran production by $S$. bovis at $37^{\circ}$ which contains no molecules larger than an amino acid and an oligosaccharide. During this work some observations have been made on the apparent need for biotin and pantothenic acid for good dextran production by some strains of $S$. bovis even under anaerobic conditions.

\section{METHODS}

Rumen strains of Streptococcus bovis. Five isolates were used of which three (2SA, $2 \mathrm{~B}$ and 18/M2) had been isolated at the Rowett Research Institute, Aberdeenshire, before 1955, and the remaining two (I and 40) at this 
laboratory quite recently (Bailey \& Oxford, 1958a). Isolates $2 \mathrm{SA}$ and 40 had previously been shown to behave a little differently from the others, in that they produced small yields of dextran in absence of added $\mathrm{CO}_{2}$.

Basal medium containing sucrose. The following was sterilized by intermittent steaming: Bacto-Casamino acids ('technical', 'certified' or 'vitaminfree' grades), 25 g.; $\mathrm{Na}$ thioglycollate, $0 \cdot 3 \mathrm{~g}$.; $\mathrm{MgSO}_{4}, 7 \mathrm{H}_{2} \mathrm{O}, 0 \cdot 2 \mathrm{~g}$.; $\mathrm{FeSO}_{4}, \mathbf{7 H}_{2} \mathrm{O}$, 0.005 g.; uracil, $0.02 \mathrm{~g}$; ; adenine sulphate, $0.05 \mathrm{~g}$.; guanine, $0.02 \mathrm{~g}$.; cystine and tryptophan, each $0 \cdot 15 \mathrm{~g}$. dissolved in a little boiling dilute sulphuric acid; Analar sucrose, 50 g.; distilled water, $1000 \mathrm{ml}$; $40 \%$ (w/v) KOH solution, a few drops to bring to $\mathrm{pH} 6$.

Additions to basal medium. Crystalline sodium acetate $(2 \mathrm{~g} . / 100 \mathrm{ml}$.) or $\mathrm{Na}_{2} \mathrm{HPO}_{4}, 12 \mathrm{H}_{2} \mathrm{O}(1.8 \mathrm{~g} . / 100 \mathrm{ml}$.) to serve as buffering agents were usually sterilized separately in the solid state in plugged 50 or $100 \mathrm{ml}$. conical flasks in which the cultures were afterwards to be grown. Alternatively, acetate (2\%) but not phosphate could be added to the basal medium before sterilization. The following sterile solutions were also added to the flasks containing buffer salts after their sterilization: $5 \%(\mathrm{w} / \mathrm{v}) \mathrm{K}_{2} \mathrm{CO}_{3}(0 \cdot 6 / 100 \mathrm{ml})$; B-vitamin solution $(1 / 100 \mathrm{ml}$.). The flasks were then filled almost full with the basal medium and the contents well mixed by a sterile glass rod. The vitamin solution contained one or more of the following eight ingredients: pyridoxine $\mathrm{HCl}$, nicotinic acid, calcium pantothenate, thiamine $\mathrm{HCl}, p$-aminobenzoic acid (each $200 \mathrm{mg} . / 1$. ); riboflavin and folic acid (each $50 \mathrm{mg} . / 1$. ); and biotin $(0.25 \mathrm{mg} . / 1$.). Another adjuvant used in conjunction with phosphate buffer only was $3 \%(\mathrm{w} / \mathrm{v})$ Bacto-tryptose before and after dialysis for $24 \mathrm{hr}$. at $2^{\circ}$. This solution was then suitably diluted and used in place of distilled water in making up the basal medium containing sucrose. The final concentration of Bacto-tryptose, or its dialysed equivalent, varied from 0.3 to $1.5 \%$ in this basal medium.

Cultural conditions. The primary inoculum for $50 \mathrm{ml}$. medium was one small loopful of a 1-day culture in Bacto-thioglycollate medium containing $1 \%(\mathrm{w} / \mathrm{v})$ sucrose. The temperature of incubation was $37^{\circ}$. Incubation was either in an almost $100 \% \mathrm{CO}_{2}$ atmosphere (see Bailey \& Oxford, 1958a) or in a metal McIntosh \& Fildes' anaerobic jar filled with a $5 \%$ (v/v) $\mathrm{CO}_{2}+95 \%(\mathrm{v} / \mathrm{v}) \mathrm{H}_{2}$ gas mixture. A tube of sterile sucrose +Bacto-thioglycollate medium containing methylene blue was also placed in the jar to indicate whether anaerobic conditions had been maintained. After 1 day the cultures were removed from the jar and when growth or dextran production was not very pronounced they were incubated for a further day in air. The addition of $\mathrm{K}_{2} \mathrm{CO}_{3}$ to the medium was to ensure the presence of some dissolved $\mathrm{CO}_{2}$, soon after lactic acid formation occurred, whatever gas mixture was above the culture.

Assessment of growth and dextran yield. When little or no dextran production occurred, growth was judged by the amount of deposit $( \pm,+$ or ++$)$ on centrifuging the culture clear. Dextran-producing cultures had first to be diluted with 2 vol. of water before centrifuging. Good dextran production $(+$ or ++$)$ was revealed by: $(a)$ a distinctly opalescent appearance; $(b)$ considerable increase in viscosity; $(c)$ difficulty in spinning down more than a 
small fraction of the organisms in an undiluted culture; $(d)$ immediate white precipitate on gentle shaking after adding ethanol (4 vol.) to the supernatant fluid from the undiluted culture (see Bailey \& Oxford, 1958a). Trace amounts of dextran, requiring prolonged shaking for separation, were ignored. The final $\mathrm{pH}$ value of the culture was also roughly determined by means of B.D.H. 4055 indicator paper.

\section{RESULTS}

\section{Acetate buffered medium}

Effect of pantothenic acid in a simple amino acid medium not specially freed from B-vitamins. Most of the preliminary work was carried out with strain I, which had been chiefly used by Bailey \& Oxford $(1958 a, b)$, and with a medium containing Bacto 'technical' or ' certified' grades of Casamino acids. The former contains more ash than the latter and there is no need to add ferrous sulphate to the medium when using it. Neither grade is guaranteed to be vitamin free; nevertheless, with use of either in conjunction with the usual procedure of omitting added B-vitamins one at a time from the total mixture of eight, it was clearly shown that good growth and dextran production took place with strain I only in those media containing added pantothenic acid. Likewise, also in $\mathrm{C}_{2}$ atmosphere, growth and good dextran production took place when pantothenate, alone among B-vitamins, was added to the basal medium containing no other added vitamins. When, however, incubation was carried out in air, the growth was always very poor and no dextran resulted even when pantothenate was added. Incubation under strict anaerobic conditions gave moderate growth, with a decrease in $\mathrm{pH}$ to $5 \cdot 2$, but no dextran with no added pantothenate, although there was good growth (final $\mathrm{pH} 4.5$ ) and good dextran production when pantothenate was added. Although therefore strain $I$ is less nutritionally exacting anaerobically than in presence of oxygen (cf. Ford et al. 1958), pantothenate as well as $\mathrm{CO}_{2}$ must always be supplied for good dextran production to occur. Presumably, the two grades of Casamino acids used are at least pantothenate-free, although adequately supplied with biotin (see below). The observation of Ford et al. (1958) was confirmed, namely, that pantothenate was not needed for good growth anaerobically when glucose was supplied in place of sucrose.

Repetition of these experiments with the other 4 strains showed that strains 18/M2 and $2 \mathrm{~B}$, but not strains 40 and $2 \mathrm{SA}$, behaved as did strain I above. The abnormal strains seemed to have less need of pantothenic acid for dextran production than the others (see Table 1). Growth, however, was usually much slower without added pantothenate. Throughout this study good growth resulted also in a decrease in $\mathrm{pH}$ value to less than 5.0. Furthermore, there was never good dextran production without good growth.

Biotin as an essential for dextran production. It was realized from the above results that nothing more than a requirement of some strains of Streptococcus bovis for pantothenate for dextran production had so far been proved. This was confirmed by use of 'vitamin-free' Casamino acids. In addition, an absolute requirement for biotin for dextran production in all 5 strains came 
to light. Thus, when to the basal medium were added the seven B-vitamins but not biotin, none of the 5 strains gave other than poor growth and no dextran under any conditions, including strict anaerobiosis. With all eight B-vitamins added, normal growth and good dextran production took place with all 5 strains, and good dextran production also occurred on the second transfer to this medium. When, however, biotin alone, or biotin + pantothenate only, were added to the basal medium, strain differences became apparent, and even as few as $\mathbf{5}$ strains fell into three distinct groups.

Thus isolates I, 18/M2 and 2B: poor growth and no dextran with biotin alone; good growth and dextran production with biotin + pantothenate.

Isolate 2SA: fair growth and dextran with biotin alone; good growth and dextran production with biotin + pantothenate.

Isolate 40: fair growth but no appreciable dextran with biotin + pantothenate; good growth and dextran production with all eight B-vitamins.

Furthermore, although growth could be quite good in a second serial transfer in the biotin + pantothenate medium, dextran production often failed to occur except in traces. For this reason it is always advisable to include the eight B-vitamins in the simplified medium when good yields of dextran are the objective. This behaviour recalls the variable behaviour towards B-vitamins of strains of Streptococcus bovis on aerobic incubation in glucose media (Ford et al. 1958). With sucrose media, however, it seemed to matter little whether incubation was under strictly anaerobic conditions or in nearly $100 \% \mathrm{CO}_{2}$ containing a little air.

\section{Phosphate buffered medium}

Effect of dialysed tryptose on dextran production. All the following experiments were carried out in a $\mathrm{CO}_{2}$ atmosphere with a basal medium containing 'technical' Casamino acids and with seven of the eight B-vitamins added, pantothenate being omitted. Whenever good growth took place in the acetatebuffered medium, there was almost always good dextran production, but the two phenomena seldom went hand in hand when phosphate was the buffering agent. From Table 1, which summarizes the results for all five isolates of Streptococcus bovis, it will be seen that only isolates 2SA and 40 gave good dextran production in the phosphate medium, although all 5 strains grew quite well. Furthermore, addition of pantothenate seemed not to be advantageous, and rather the reverse with isolate $2 \mathrm{SA}$. Isolates I and 18/M2 which grew well without dextran production in simple phosphate media, produced dextran in similar media containing a high concentration $(1.5 \%$, w/v) of tryptose either dialysed or undialysed, provided that pantothenate was also supplied. Further trials showed that a smaller concentration of tryptose $(0 \cdot 3-1 \cdot 2 \%, w / v)$ had little effect in inducing dextran formation. No essential change in the properties of the strain had occurred in those instances where good growth without dextran production took place, since plating on to $3 \%(w / v)$ sucrose $+3 \%(w / v)$ Bacto-proteose peptone agar and subsequent incubation in $100 \% \mathrm{CO}_{4}$ gave the usual large mucoid colonies typical of $S$. bovis under these conditions (cf. Bailey \& Oxford, 1958a). 


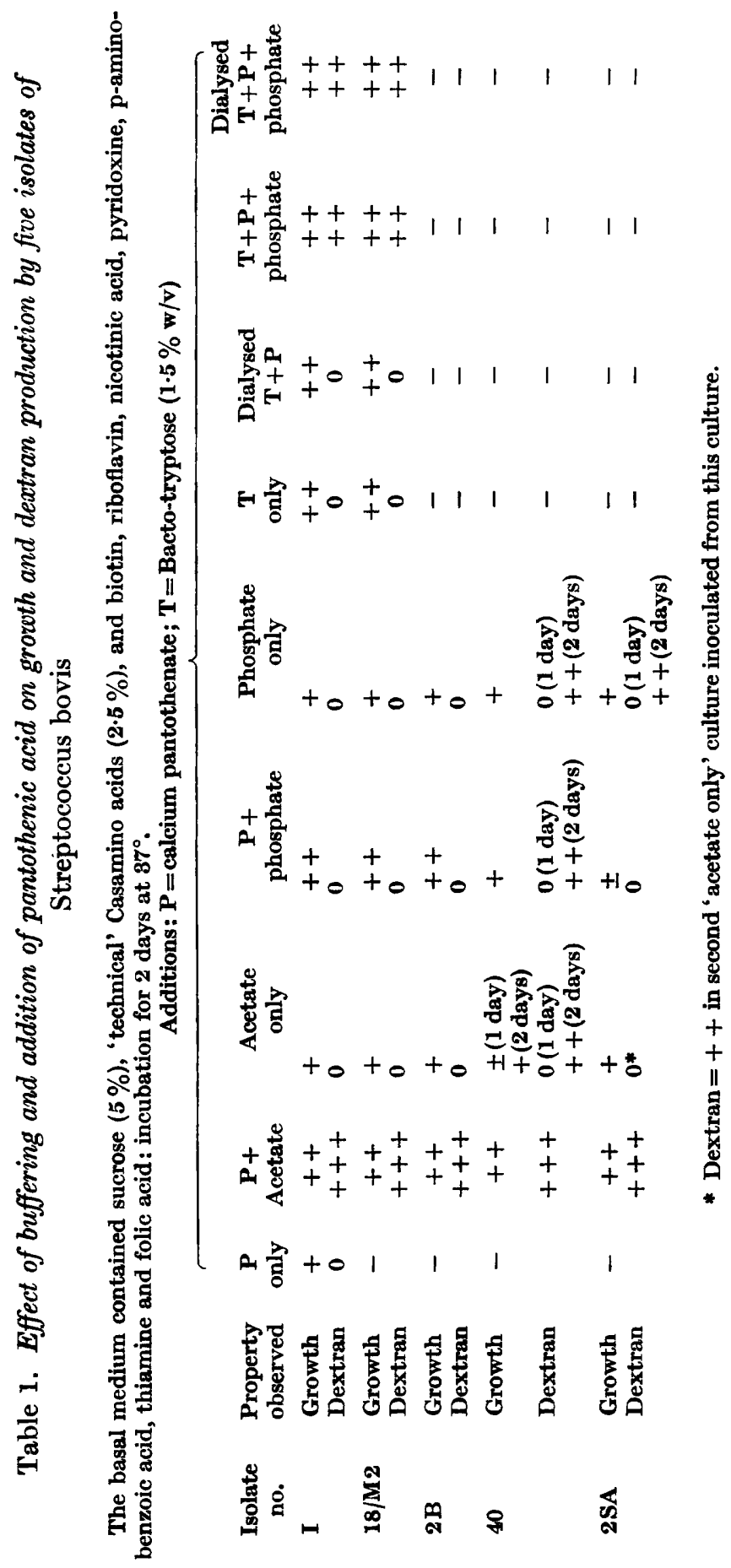


Niven et al. (1948) found that their strains of Streptococcus bovis would grow in a simple sucrose + phosphate medium containing thiamine, pantothenate, nicotinic acid, biotin, arginine and glutamic acid. None of the five isolates of $S$. bovis used in the present investigation grew in this medium. Unlike ours, all the strains of Niven et al. fermented mannitol and in this and other respects were atypical, although apparently they could produce dextran.

\section{DISCUSSION}

The fact that rumen strains of Streptococcus bovis seem always to require biotin, and often pantothenate and perhaps other B-vitamins as well, for good anaerobic production of dextran from sucrose, yet will grow well anaerobically without any B-vitamins in a glucose medium (Ford et al. 1958) is another instance of the dependence of microbial B-vitamin requirements upon the task set the micro-organism (see also observations on Leuconostoc below). There is no evidence, however, to suggest that either of these vitamins is directly concerned in the production of the enzyme dextransucrase; it would seem rather that this enzyme can be produced in quantity only when growth of the organism is rapid and profuse, and this does not occur in sucrose media without the addition of B-vitamins. In this connexion it is instructive to compare the vitamin requirements of $S$. bovis with those of Leuconostoc dextranicum as reported by Whiteside-Carlson \& Rosano (1951). The last-named organism is more exacting, since for full growth in sucrose media it required five B-vitamins, namely, nicotinic acid, thiamine, pantothenic acid, folic acid (or $p$-aminobenzoic acid) and either pyridoxal or pyridoxamine. Strangely, $L$. dextranicum does not require added biotin for growth or dextran production in sucrose media, although it does require added biotin for growth in glucose media. It differs, however, from $S$. bovis in not having a $\mathrm{CO}_{2}$ requirement for dextran production (see Bailey \& Oxford, 1958b).

In view of the earlier work of Bailey \& Oxford (1958a) who grew Streptococcus bovis for dextran production in a phosphate-buffered medium (containing also yeast extract and Casitone), the finding that this organism can grow well in a simple sucrose + amino acid + phosphate medium of high buffer capacity in presence of ample $\mathrm{CO}_{2}$ and yet produce no dextran was very surprising. The explanation for this behaviour is probably that, unlike acetate, phosphate does not act as a buffer in the narrow $\mathrm{pH}$ range over which dextransucrase is secreted into the culture, which is always tending to become more acid as a result of lactic-acid production. According to Dr R. W. Bailey (to be published)

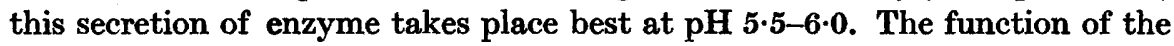
soluble protein (non-dialysable) fraction of Bacto-tryptose in stimulating dextran synthesis in a phosphate-buffered medium is not yet fully understood. It may merely help to stabilize what little enzyme is secreted under these conditions. There can be no doubt that acetate is the buffer of choice in growing $S$. bovis for dextran production: it has hardly ever been observed to fail in presence of $\mathrm{CO}_{2}$ and the appropriate $\mathrm{B}$-vitamins.

Since it is all too easy to grow known dextran-synthesizing strains of Strepto- 
coccus bovis in a sucrose medium and observe no dextran production, it is pertinent to inquire whether dextran formation is commoner among streptococci, especially of the viridans group, than the literature might lead one to suppose. The following statements are culled from the latest (7th) edition of Bergey's Manual (1957).

Viridans group. Streptococcus salivarius produces both levan and dextran from sucrose. $S$. bovis: 'some strains synthesize a dextran'. S. mitis: 'only rare strains produce large mucoid colonies on $5 \%$ sucrose agar'. $S$. equinus, $S$. thermophilus and $S$. uberis: 'no polysaccharide synthesized from sucrose'. Since the work of Dain, Neal \& Seeley (1956) and of Bailey \& Oxford (1958a) has shown that the above comment on $S$. bovis is an understatement, it might be worthwhile to re-investigate the last four viridans streptococci mentioned, with the use of techniques of buffering, vitamin addition and $\mathrm{CO}_{2}$ incubation as in this present investigation. Furthermore, there may be hitherto unsuspected cases of possible dextran production among many groups of streptococci and related bacteria since $\boldsymbol{S}$. sanguis (Group $\mathbf{H}$ ) is known to produce dextran.

I am indebted to Dr R. W. Bailey for preparing the dialysed tryptose and also for many helpful discussions.

\section{REFERENCES}

Batley, R. W. \& Oxford, A. E. (1958a). A quantitative study of the production of dextran from sucrose by rumen strains of Streptococcus bovis. J. gen. Microbiol. $19,130$.

Bailey, R.JW. \& Oxford, A. E. (1958b). Pre-requisites for dextran production by Streptococcus bovis. Nature, Lond. 182, 185.

Bailey, R. W., Barker, S. A., Bourne, E. J. \& Stacey, M. (1957). Immunopolysaccharides. Part VI. The isolation and properties of the dextransucrase of Betacoccus arabinosaceous. J. chem. Soc. p. 3530.

Bergey's Manual of Determinative Bacteriology (1957). 7th ed. Ed. by Breed, R. S., Murray, E. G. D. \& Smith, N. R. Baltimore: The Williams \& Wilkins Company.

Dain, J. A., Neal, A. L. \& Seeley, H. W. (1956). The effect of carbon dioxide on polysaccharide production by Streptococcus bovis. J. Bact. 72, 209.

Ford, J. E., Perry, K. D. \& Briggs, C. A. E. (1958). Nutrition of lactic acid bacteria isolated from the rumen. J. gen. Microbiol. 18, 273.

Niven, C. F., Washburn, M. R. \& White, J. C. (1948). Nutrition of Streptococcus bovis. J. Bact. 55, 601.

Whiteside-Carlson, V. \& Rosano, C. L. (1951). Nutritional requirements of Leuconostoc dextranicum for growth and dextran synthesis. J. Bact. 62, 583. 